\title{
iccafd
}

Revista Iberoamericana de Ciencias de la Actividad Fisica y el Deporte

\section{PRESCRIPCIÓN DEL EJERCICIO FÍSICO TERAPÉUTICO SUPERVISADO EN LA CERVICALGIA MECÁNICA INESPECÍFICA}

\section{SUPERVISED PHYSICAL THERAPEUTIC EXERCISE'S PRESCRIPTION IN THE UNSPECIFIC MECHANICAL NECK PAIN}

Albornoz Cabello, Manuel (*), Barrios Quinta, Cristo Jesús (**)

Barrios Quinta, Ana María (**) y Cruz Torres, Blanca de la (*)

D. Manuel Albornoz Cabello. (*) malbornoz@us.es

D. Cristo Jesús Barrios Quinta. ${ }^{* *}$ ) cristoj.barrios.sspa@juntadeandalucia.es

Da Ana María Barrios Quinta. (**) anam.barrios.sspa@juntadeandalucia.es

Da Blanca de la Cruz Torres. (*) bcruz@us.es

* Departamento de Fisioterapia de la Universidad de Sevilla.

** Servicio Andaluz de Salud (SAS)

Correspondencia:

D. Cristo Jesús Barrios Quinta.

Código UNESCO: 321311

DOI: http://dx.doi.org/10.24310/riccafd.2018.v7i2.5091

\section{RESUMEN:}

La cervicalgia mecánica $(\mathrm{CM})$ es una afección muy prevalente que provoca dolor e incapacidad y representa un gran coste económico, social y laboral.

El objetivo fue evaluar la eficacia de un programa de ejercicio físico terapéutico (EFT) supervisado en la reducción del dolor y la discapacidad de pacientes con CM.

Sobre una muestra de 21 pacientes con CM, de ambos sexos, pertenecientes al Servicio de Fisioterapia del Centro de Salud de San José de la Rinconada (Sevilla). Los sujetos se evaluaron al inicio y al final de la intervención (2 semanas y 10 sesiones de tratamiento). Analizamos el dolor percibido: Escala Visual Analógica (EVA), el grado de discapacidad: Índice de Discapacidad del Cuello (NDI) y Core Outcome Measure (COM).

El programa de EFT en pacientes con CM produjo una reducción del dolor VAS $=3,74+/-1,33$ y de la discapacidad $\mathrm{NDI}=16,28+/ 4,97$ y $\mathrm{COM}=21,69+/ 10,54$, de forma estadísticamente significativa $(p=0,000$ para $p<0,05)$.

El programa de EFT presentado fue eficaz en la reducción del dolor y la discapacidad en los pacientes con CM.

Palabras clave: ejercicio físico terapéutico, cervicalgia mecánica, fisioterapia. 


\section{ABSTRACT:}

Mechanical cervicalgia (CM) is a very prevalent condition that causes pain and disability and represents a great economic, social and labor cost.

The objective was to evaluate the efficacy of a supervised therapeutic physical exercise program (EFT) in reducing pain and disability in CM patients.

A sample of 21 patients with CM, of both sexes, belonging to the Physical Therapy Service of the Health Center of San José de la Rinconada (Seville). Subjects were assessed at the beginning and at the end of the intervention (2 weeks and 10 treatment sessions). We analyze the perceived pain: Visual Analogue Scale (EVA), the degree of disability: Neck Disability Index (NDI) and Core Outcome Measure (COM).

The EFT program in patients with CM produced a reduction in pain VAS $=3.74$ $+/-1.33$ and disability NDI $=16.28+/ 4.97$ and $\mathrm{COM}=21.69+/ 10.54$, in a statistically significant way ( $p=0.000$ for $p<0.05$ ).

The EFT program presented was effective in reducing pain and disability in CM patients.

Key words: therapeutic physical exercise, mechanical cervicalgia, physiotherapy

\section{INTRODUCCIÓN}

Las algias músculo-esqueléticas de la columna vertebral más frecuentes se localizan a nivel cervical y lumbar. Así, el dolor cervical presenta una prevalencia de un $25-30 \%$ y el dolor lumbar se sitúa entre el $22-65 \%$, en la población en general (1).

Según la Encuesta Nacional de Salud (ENS, 2014) más de dos millones de personas entre 16 y 64 años consideran sus problemas de espalda o dolor de cuello como la dolencia más importante y que más afecta a su calidad de vida. Si distinguimos la prevalencia por sexos, la cervicalgia afecta de forma crónica en un $23,9 \%$ a los varones y en un $29,2 \%$ a las mujeres (1). Podemos concluir en base a estos datos, de la Encuesta Europea de Salud del año 2014, que nos encontramos frente al $5^{a}$ problema de salud crónico en porcentaje de población afectada en España (5).

Si diferenciamos el riesgo de padecer dolor cervical de origen inespecífico, o cervicalgia mecánica (CM), en función de la actividad laboral desempeñada, la prevalencia anual marca valores entre un $12.1 \%$ a un $75.5 \%$ en la población general y de un $27.1 \%$ a un $47.8 \%$ para la población trabajadora, siendo considerada una de las primeras causas de absentismo laboral. (2)

Basándonos en las publicaciones más recientes sobre la base de la cartera de servicios de la Fisioterapia en Atención Primaria, se identificó que el 95,45\% de los motivos de derivaciones correspondían a enfermedades músculo-esqueléticas no traumáticas. Resulta destacable que el $19,55 \%$ de los motivos de derivaciones estaba constituido por síndromes relacionados con la columna cervical y 
el 17,27\% eran síndromes del hombro (3).Actualmente, la cervicalgia constituye la segunda causa de consulta más frecuente en Atención Primaria (AP), seguida de la lumbalgia (4). La incidencia anual de la cervicalgia en AP se calcula en valores de 12 por cada 1000 habitantes (5).

El manejo del dolor músculo-esquelético es uno de los más importantes retos que afrontan los fisioterapeutas en su práctica clínica diaria. Los costes sanitarios que genera la cervicalgia son importantes. Sin embargo, el volumen más importante procede de los costes de compensación por bajas en el ámbito laboral, donde se ha llegado a comprobar que causan tantas bajas como la lumbalgia (6).Puede afirmarse, sin temor a exagerar, que en los países desarrollados existe una auténtica epidemia, pese a que se dispone de un conocimiento mayor de los mecanismos neurofisiológicos del dolor y de mejores herramientas terapéuticas para tratarlo (7).

Tradicionalmente, se ha considerado a la Fisioterapia como una intervención secundaria, dirigida al restablecimiento de la función una vez finalizado el tratamiento de la patología. Esto ha llevado a que el paciente fuera derivado al fisioterapeuta muy tardíamente lo que limitaba las posibilidades de un tratamiento eficaz. Actualmente, muchas recomendaciones y guías clínicas consideran que la Fisioterapia juega un papel central en el tratamiento del dolor y en la prevención de la discapacidad crónica relacionada con el dolor $(8,9)$. Sin embargo, no existe un consenso entre los profesionales de la salud del papel que puede jugar la Fisioterapia en el manejo de los pacientes con dolor crónico.

El ejercicio físico terapéutico (EFT) y la integración de la educación sanitaria individual y en grupo, en la actividad del fisioterapeuta de AP, depara a medio plazo un mayor control de las demandas recidivantes en fisioterapia (10). Por ello el EFT supervisado se ha postulado como una de las herramientas más eficaces en la resolución de problemas derivados de las afecciones crónicas donde el dolor y la discapacidad son los objetivos principales a conseguir $(10,11)$

El objetivo del estudio fue evaluar la eficacia de un programa de ejercicio físico terapéutico (EFT) supervisado en la reducción del dolor y la discapacidad de pacientes con cervicalgia mecánica $(\mathrm{CM})$ en atención primaria medidos por medio de la escala visual analógica y el Índice de discapacidad del cuello (NDI) y el Core Outcome Measure (COM).

\section{MÉTODO}

El presente estudio es longitudinal y prospectivo, sin grupo control, llevado a cabo en el Servicio de Fisioterapia del Centro de Salud de San José de La Rinconada (Sevilla), dependiente del Servicio Andaluz de Salud.

La población diana a la que va dirigido este protocolo estuvo formada por todos los pacientes con diagnóstico de cervicalgia mecánica (CM) de la Zona Básica de Salud de La Rinconada de Sevilla, con más de 36.000 usuarios po- 
tenciales.

La muestra del estudio se seleccionó de forma intencionada de entre los pacientes que acudieron a las consultas médicas de Atención Primaria (AP) de los Centros de Salud de San José de La Rinconada de Sevilla y que siguieron los siguientes criterios de inclusión y exclusión.

Los criterios de inclusión establecidos fueron: estar diagnosticados cervicalgia mecánica; tener una edad mayor de 55 años y dar el consentimiento para la realización del estudio.

Los criterios de exclusión establecidos fueron: haber sido intervenidos quirúrgicamente a nivel de columna vertebral; presentar cefaleas de origen no cervicogénico; padecer vértigo de origen no cervicogénico; sufrir cervicalgia asociada a déficit neurológico importante (alteración de los reflejos, espasticidad); presentar fiebre inexplicable durante el estudio y padecer diagnósticos específicos (estenosis cervical, osteomielitis cervical, mielopatías).

Previamente al estudio de campo desarrollado se solicitó a todos los sujetos del estudio un consentimiento informado de acuerdo con la declaración de Helsinki, en su última revisión de octubre de 2004, tras ser informados en detalle sobre la investigación a realizar y conforme a las modificaciones pertinentes realizadas siguiendo las indicaciones del comité de Investigación de los Hospitales Virgen Macarena y Virgen del Rocío y con su dictamen favorable https://www. juntadeandalucia.es/salud/portaldeetica/xhtml/ayuda/verificarFirmaDocu mento. iface/code/03abac9cead37366ec394b2702b95ee21b4b99df.

Las mediciones de las distintas variables del estudio se hicieron en dos momentos, al comienzo del mismo y transcurrido un periodo 2 semanas, que coincidió con el fin de la intervención o plan de tratamiento (10 sesiones de EFT supervisado).

Las variables del estudio fueron: la percepción del dolor cervical medido con la Escala Visual Analógica (EVA). Esta variable se ha obtenido mediante una escala validada, de 10 centímetros (0-10), donde el sujeto se auto-posicionó desde ausencia de dolor (0) hasta el peor dolor soportable (10) (12). El grado de incapacidad de los pacientes se ha recogido a través de la Escala de Incapacidad por Dolor Cervical o Neck Disability Index (NDI) con un máximo de 50 puntos. (11-14). La puntuación puede multiplicarse x2 para expresarse en un porcentaje (\%). Podemos hablar de una discapacidad leve si está comprendida entre 10$28 \%$; moderada de $30-48 \%$; severa $50-64 \%$ e incapacidad completa $70-100 \%$ (17). Finalmente, al discapacidad fue evaluada a través del cuestionario Core Outcome Measure (COM) (15-17). Este cuestionario está validado y es específico para el estudio de la discapacidad y de la calidad de vida de pacientes con cervicalgia. En ella se le solicita al sujeto experimental que se auto-posicione en diferentes dimensiones de su vida diaria.

El protocolo de intervención con EFT supervisado se basó en un programa de ejercicios de fisioterapia activa para los músculos del cuello y del hombro, siguiendo las directrices del manual: «Pautas de Actuación Conjunta de los Equi- 
pos de Básicos de Atención Primaria y los Equipos de Apoyo a la Rehabilitación». (18). Dicho programa de ejercicios incluye: ejercicios de estiramiento activo y ejercicios de fortalecimiento muscular isométrico. Además, se realizó un programa de reeducación óculo cérvico cinética (figura $\mathrm{n}^{0} 1$ ). El control de la realización de los ejercicios propuestos se llevó a cabo mediante un diario que los pacientes debían rellenar en su domicilio.
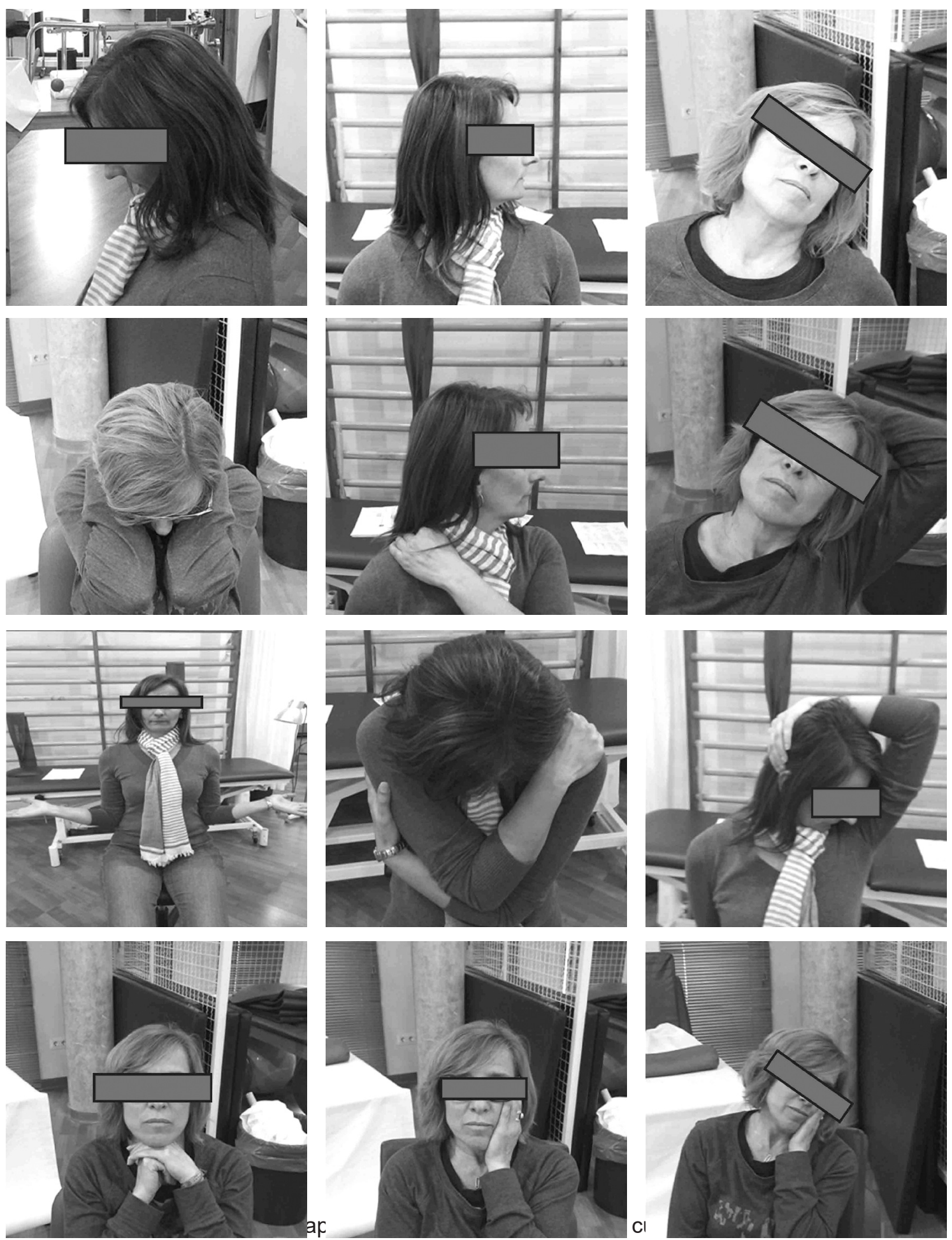

ANÁLISIS ESTADÍSTICO 
Se realizó un análisis descriptivo de cada una de las variables, mostrando sus valores medios \pm desviaciones típicas. Para conocer si la muestra seguía una distribución normal se realizó el test de Shapiro-Wilks. Tras determinar que la muestra seguía una distribución normal para la variable dolor percibido y discapacidad, se compararon los valores obtenidos para cada variable en los diferentes momentos de valoración mediante el test de la t de Student para muestras apareadas, de manera respectiva. Se calculó el Indice de correlación intraclase (ICC) con un intervalo de confianza del $95 \%$ para conocer la validez de las mediciones realizadas. El tamaño de efecto clínico se analizó mediante la d de Cohen, cuyos valores son $>0.8$ son considerado grande, de 0.5 moderado y bajo $<0.2$. El nivel de significación se estableció en un valor de $p<0,05$. El análisis de los datos se realizó mediante el software estadístico SPSS, versión 19.0.

\section{RESULTADOS}

La muestra está constituida por 21 sujetos, 8 hombres y 13 mujeres, con una media de edad de 56 $\pm 4,33$ años de edad [55-65], de los que 11 tenían estudios primarios 9 estudios secundarios y 1 era universitario. El índice de masa corporal (IMC) medio era de 27,94 $\pm 5,77$ y una mediana de 25,2; por lo que estamos ante un $50 \%$ de la muestra que presenta sobrepeso, del cual el $25 \%$ son obesos.

El 57\% (12/21) de los pacientes estaban bajo tratamiento farmacológico con analgésicos y antinflamatorios no esteroideos y el 43\% (9/21) no tomaban ningún medicamento.

Los sujetos muestrales trabajan en el sector servicios en un 57\% (12/21) y el resto se distribuían entre trabajos por cuenta ajena (2/21), en la construcción $(2 / 21)$ y 5 sujetos eran pensionistas o parados.

Como se observa en la tabla $n^{\circ} 1$ los valores medios de las variables que miden el dolor y la discapacidad; así como las correlaciones de los mismos nos informan de la validez del proceso de medición.

Tabla 1. Valores medios e índice de correlación intraclase (ICC) del dolor y la discapacidad antes

\begin{tabular}{|c|c|c|c|c|c|}
\hline & Media & $\begin{array}{c}\text { Desviación } \\
\text { estándar }\end{array}$ & ICC & IC 95\% & $p$ \\
\hline EVA pre & 6,82 & 1,306 & \multirow{2}{*}{,661 } & \multirow{2}{*}{,165-,863 } & \multirow{2}{*}{,010 } \\
\hline EVA post & 3,08 & 1,350 & & & \\
\hline NDI pre & 28,24 & 6,602 & \multirow{2}{*}{,77 } & \multirow{2}{*}{ 433-,907, } & \multirow{2}{*}{,001 } \\
\hline NDI post & 11,95 & 4,738 & & & \\
\hline COM pre & 42,35 & 11,620 & \multirow{2}{*}{ 60 } & \multirow{2}{*}{,08-,837 } & \multirow{2}{*}{,024 } \\
\hline COM post & 20,66 & 7,650 & & & \\
\hline
\end{tabular}

(pre) y después (post) del tratamiento. 


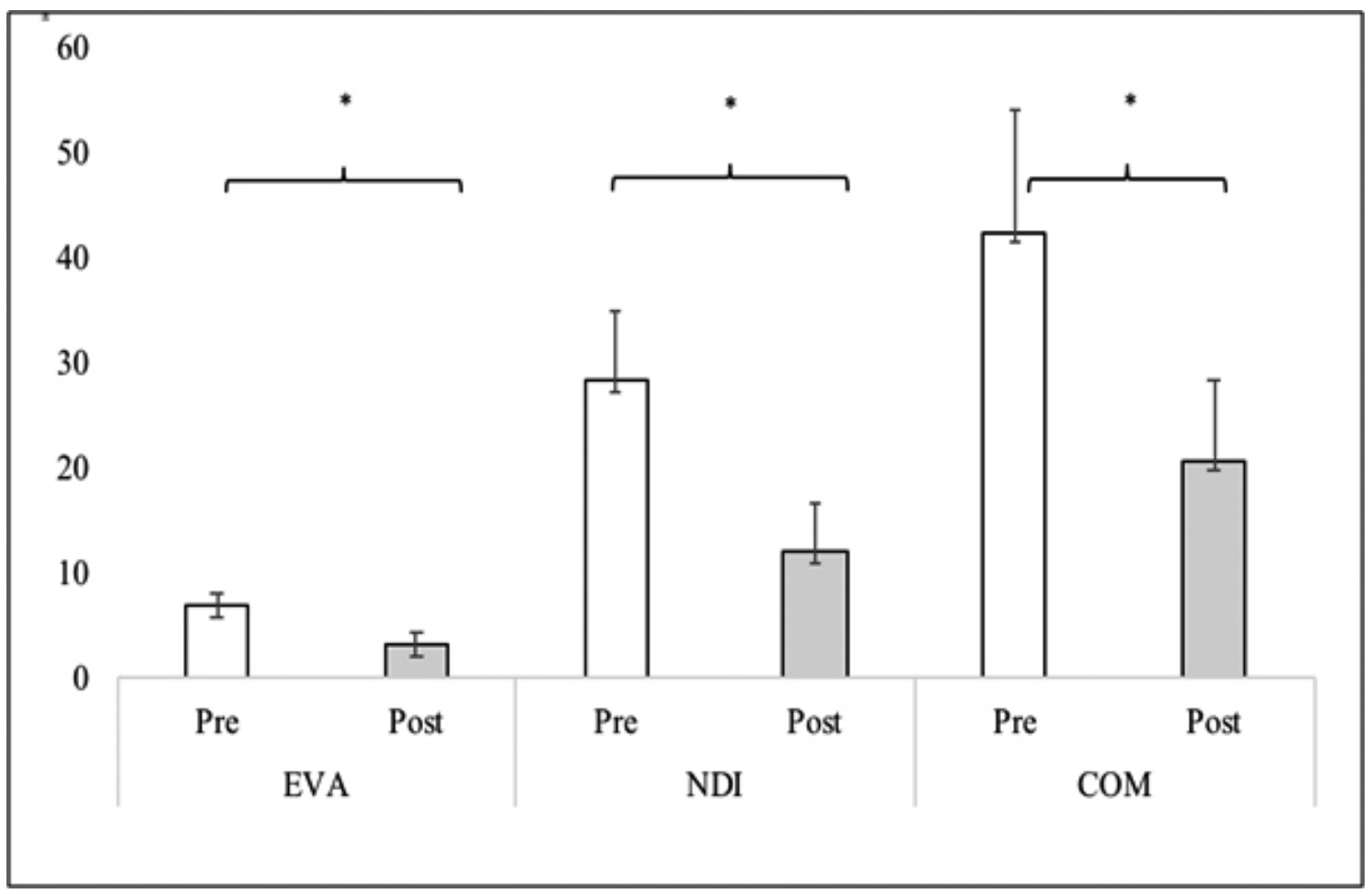

Figura 2. Reducción de los valores medios de EVA, NDI Y COM del momento inicial al final del estudio.

Como se muestra en la tabla no: 2 el análisis efectuado mediante el estadístico de la $\mathrm{T}$ de Student para muestras emparejadas, de las variables dolor, medido a través de la escala EVA, y la discapacidad evaluada a través del NDI y el COM, antes (pre) y después (post) del tratamiento, nos refleja que existen cambios estadísticamente significativos $(p=0,000$ para $p<0,05)$ en todas las variables analizadas. Además, se ha calculado el tamaño del Efecto Clínico a través del estadístico d de Cohen obteniéndose un tamaño de efecto grande ya que $>0.8$ aproximadamente un 0,96 . Cabe destacar la reducción de 3,74 puntos en la escala EVA pasando de un valor medio 6,82/10 a un 3,08/10 después del tratamiento.

En el caso de la discapacidad medida a través del NDI se ha pasado de una puntuación de 28,24/50 a un 11,95/50. Que, expresado en porcentaje, inicialmente representa un $56 \%$ o lo que es lo mismo una discapacidad severa, pasando después del tratamiento a un 24\%; es decir, una discapacidad leve. Además, se ha calculado el tamaño del Efecto Clínico a través del estadístico d de Cohen obteniéndose un tamaño de efecto grande ya que $>0.8$ aproximadamente un 0,95. Cabe destacar la reducción media de 16,28 puntos pasando de 28,24 a 11,95 . Presenta además esta diferencia significación estadística ( $p=0,000$ para $p<0,05)$.

En relación con el COM nos confirma lo expresado anteriormente que el protocolo de EFT supervisado provoca en pacientes con CM una reducción de la discapacidad del $51 \%$. Además, se ha calculado el tamaño del Efecto Clínico a 
través del estadístico d de Cohen obteniéndose un tamaño de efecto grande ya que $>0.8$ aproximadamente un 0,90. Cabe destacar la reducción media de 21,69 puntos pasando de 42,35 a 20,66. Además, podemos afirmar que estos cambios alcanzan significación estadística $(p=0,000$ para $p<0,05)$.

\begin{tabular}{|c|c|c|c|c|c|c|}
\hline & Media & $\begin{array}{l}\text { Desviación } \\
\text { estándar }\end{array}$ & $\begin{array}{l}95 \% \text { de } \\
\text { confianz } \\
\text { cia }\end{array}$ & $\begin{array}{l}\text { valo de } \\
\text { la diferen- }\end{array}$ & $\mathrm{p}$ & $d$ \\
\hline & & & Inferior & Superior & & \\
\hline EVA pre-post & 3,74 & 1,336 & 3,13 & 4,35 & ,000 & 0,96 \\
\hline NDI pre-post & 16,28 & 4,971 & 14,02 & 18,54 & ,000 & 0,95 \\
\hline COM pre-post & 21,69 & 10,542 & 16,89 & 26,48 & ,000 & 0,90 \\
\hline
\end{tabular}

Tabla 2. T de Student para muestras emparejadas del dolor y la discapacidad antes (pre) y después (post) del tratamiento.

\section{DISCUSIÓN}

El ejercicio terapéutico es ampliamente usado y recomendado en el tratamiento del dolor cervical crónico inespecífico con el objetivo de mejorar el dolor y la discapacidad relacionada, mejorar la función músculo-esquelética, la satisfacción del paciente y la calidad de vida (19-28)

En relación con las diferentes Guías de Práctica Clínica, destacar que la Philadephia Panels ya señala el beneficio clínico incrementado del ejercicio físico terapéutico en comparación a otras modalidades terapéuticas tales como el ultrasonido, la termoterapia o el masaje. (29) Los estudios desarrollados por Nachemson et al. También demuestran la existencia de una evidencia moderada a favor del empleo del ejercicio terapéutico en el abordaje de la cervicalgia tanto en su fase aguda como crónica. (30)

En relación a los estudios desarrollados sobre las Escuelas de Espalda (EE) los resultados publicados hasta la fecha son bastante dispares en parte por la difícil comparación entre estudios dadas las diferentes variables tenidas en cuenta en su desarrollo como por la calidad metodológica de estos. Parece concluirse que la efectividad de dichos programas va a depender de la severidad de la sintomatología, así como de diversos factores de riesgo añadidos como puede ser las cargas físicas del desempeño laboral de los sujetos. $(31,32)$

No obstante, no hay acuerdo sobre los protocolos a usar y los programas de ejercicio terapéutico para el dolor cervical varían en cuanto al tipo de ejercicio (general vs específico, de resistencia vs movilidad), y a las modalidades de entrenamiento (intensidad, volumen, orden del ejercicio, frecuencia, duración, intervalos de descanso). Los protocolos usados están basados sobre ejercicios de fuerza, ejercicios propioceptivos y de fijación ocular (33), de coordinación y 
de equilibrio, ejercicios de reeducación postural y de estiramiento muscular. La eficacia de programas de ejercicios de relajación no parece comprobada (34). La terapia cognitivo conductual planteada en los estudios de Lipton sí parecen resultar de utilidad en el abordaje de los pacientes aquejados de cervicalgia demostrando una disminución en los niveles de dolor, mejora en la funcionalidad, la calidad de vida e incluso reduciendo el consumo de fármacos.(2)

La mayor parte de los estudios concluyen que existe una evidencia insuficiente en relación los tipos o dosificación delos ejercicios terapéuticos en el manejo de los pacientes aquejados de cervicalgia mecánica y apuntan a esa línea de investigación como esencial para los futuros estudios.

Uno de los enfoques más impulsados en el tiempo reciente por las direcciones de las unidades de gestión clínica sanitaria para el manejo de la cervicalgia mecánica inespecífica es aquel que pone el énfasis en el autocuidado del paciente y la gestión eficiente de recursos profesionales del sistema sanitario a través del empleo de la combinación de ejercicio terapéutico supervisado y consejo ergonómico. En las últimas revisiones Cochrane, no se ha podido constatar una evidencia clara (35), por lo que sería una línea muy interesante a desarrollar en atención primaria; más teniendo en cuenta, que, al igual que consideran muchos fisioterapeutas las recomendaciones de higiene postural y el ejercicio terapéutico debieran ser la piedra angular de la fisioterapia en nuestro ámbito.

\section{CONCLUSIONES}

El programa de ejercicio físico terapéutico supervisado propuesto es válido y fiable en la reducción del dolor y el grado de discapacidad de los pacientes aquejados de cervicalgia mecánica inespecífica

El programa de ejercicio físico terapéutico supervisado propuesto reduce el dolor percibido en pacientes con cervicalgia mecánica medido a través de la escala visual analógica de forma estadística y clínicamente significativa.

El programa de ejercicio físico terapéutico supervisado propuesto disminuye el grado de discapacidad de los pacientes aquejados de cervicalgia mecánica inespecífica medido a través del Índice de Discapacidad del Cuello de forma estadística y clínicamente significativa.

El programa de ejercicio físico terapéutico supervisado propuesto reduce el grado de discapacidad de los pacientes aquejados de cervicalgia mecánica inespecífica medido a través del cuestionario Core Outcome Measure de forma estadística y clínicamente significativa. 


\section{REFERENCIAS}

1. Europea E. 30 de noviembre de 2010 Encuesta Europea de Salud en España . Año 2009 Principales resultados Problemas crónicos de salud Autonomía personal. 2011;1-8.

2. Calahorrano-Soriano C, Abril-Carreres A, Quintana S, Permanyer-Casals E, GarretaFiguera R. Programa rehabilitador integral del raquis cervical. Descripción, resultados y análisis de costes. Rehabilitacion [Internet]. 2010 [cited 2016 Jun 10];44(3):20510.

3. Rebelo Ferreira de Carvalho S, Vilella Bueno S, Martin Casas P, Bonilla Solís R. Fisioterapia en las unidades de apoyo de los Equipos de Atención Primaria de Salud de la Comunidad de Madrid. Fisioterapia [Internet]. 2014 [cited 2016 Oct 26];36(2):81-6.

4. Escortell Mayor E, Lebrijo Pérez G, Pérez Martín Y, Asúnsolo del Barco Á, Riesgo Fuertes R, Saa Requejo C. Ensayo clínico aleatorizado en pacientes con cervicalgia mecánica en atención primaria: terapia manual frente a electroestimulación nerviosa transcutánea. Atención Primaria [Internet]. 2008;40(7):337-43.

5. Walker BF. The prevalence of low back pain: a systematic review of the literature from 1966 to 1998. J Spinal Disord [Internet]. 2000 Jun [cited 2016 Oct 10];13(3):205-17.

6. Borghouts JA, Koes BW, Vondeling H, Bouter LM. Cost-of-illness of neck pain in The Netherlands in 1996. Pain [Internet]. 1999 Apr [cited 2016 Jun 4];80(3):629-36.

7. Loeser JD. What is chronic pain? Theor Med [Internet]. 1991 Sep [cited 2016 Jun 4];12(3):213-25.

8. Goode AP, Freburger J, Carey T, Carolina N, Hill C. Prevalence, Practice Patterns, and Evidence for Chronic Neck Pain. 2010;62(11):1594-601.

9. Kroeling P, Gross AR, Goldsmith CH. A Cochrane Review of Electrotherapy for Mechanical Neck Disorders. 2005;30(21):641-8.

10. Medina Mirapeix F, Brotons Roman J, Manrique Sánchez J. [Comparative study on the influence of health education on perceived recurrences after physiotherapy]. Aten primaria / Soc Española Med Fam y Comunitaria [Internet]. 1995 Nov 15 [cited 2016 Jun 18];16(8):464-8.

11. Cleland JA, Fritz JM, Whitman JM, Palmer JA. The reliability and construct validity of the Neck Disability Index and patient specific functional scale in patients with cervical radiculopathy. Spine (Phila Pa 1976) [Internet]. 2006 Mar 1 [cited 2016 Jun 20];31(5):598-602.

12. Vernon H, Mior S. The Neck Disability Index: a study of reliability and validity. J Manipulative Physiol Ther [Internet]. 1991 Sep [cited 2016 Jun 20];14(7):409-15.

13. Cleland JA, Childs JD, Whitman JM. Psychometric Properties of the Neck Disability Index and Numeric Pain Rating Scale in Patients With Mechanical Neck Pain. Arch Phys Med Rehabil [Internet]. 2008 Jan [cited 2016 Jun 18];89(1):69-74.

14. MacDermid JC, Walton DM, Avery S, Blanchard A, Etruw E, McAlpine C, et al. Measurement properties of the neck disability index: a systematic review. J Orthop Sports Phys Ther [Internet]. 2009 May [cited 2016 Jun 18];39(5):400-17. 5

15. White $P$, Lewith $G$, Prescott $P$. The core outcomes for neck pain: validation of a new outcome measure. Spine (Phila Pa 1976) [Internet]. 2004 Sep 1 [cited 2016 Oct 11];29(17):1923-30. 
16. Murphy DR, Lopez M. Neck and back pain specific outcome assessment questionnaires in the Spanish language: a systematic literature review. Spine J. 2013;13(11):1667-74.

17. Fankhauser $C D$, Mutter U, Aghayev E, Mannion AF. Validity and responsiveness of the Core Outcome Measures Index (COMI) for the neck. Eur Spine J [Internet]. 2012 Jan [cited 2016 Oct 8];21(1):101-14.

18. Servicio Andaluz de Salud. Pautas de Actuación Conjunta de los Equipos de Básicos de Atención Primaria y los Equipos de Apoyo a la Rehabilitación. 2006;

19. Gross AR, Paquin JP, Blanchette S, Lalonde P, Christie T, Dupont G, et al. Exercise for mechanical neck disorders: A cochrane systematic review update. Physiother (United Kingdom) [Internet]. 2015;101:eS486.

20. Andersen LL, Kjaer M, Søgaard K, Hansen L, Kryger Al, Sjøgaard G. Effect of two contrasting types of physical exercise on chronic neck muscle pain. Arthritis Rheum [Internet]. 2008 Jan 15 [cited 2016 Jun 19];59(1):84-91.

21. Escolar-Reina P, Medina-Mirapeix F, Gascón-Cánovas JJ, Montilla-Herrador J, Jimeno-Serrano FJ, de Oliveira Sousa SL, et al. How do care-provider and home exercise program characteristics affect patient adherence in chronic neck and back pain: a qualitative study. BMC Health Serv Res [Internet]. 2010 [cited 2016 Jun 4];10:60.

22. O'Riordan C, Clifford A, Van De Ven P, Nelson J. Chronic neck pain and exercise interventions: Frequency, intensity, time, and type principle. Arch Phys Med Rehabil [Internet]. 2014;95(4):770-83.

23. Jull G, Falla D, Treleaven J, Hodges P, Vicenzino B. Retraining cervical joint position sense: the effect of two exercise regimes. J Orthop Res [Internet]. 2007 Mar [cited 2016 Jun 19];25(3):404-12.

24. Miller J, Gross A, D'Sylva J, Burnie SJ, Goldsmith CH, Graham N, et al. Manual therapy and exercise for neck pain: A systematic review. Man Ther [Internet]. 2010;15(4):334-54.

25. Chiu TTW, Lam T-H, Hedley AJ. A randomized controlled trial on the efficacy of exercise for patients with chronic neck pain. Spine (Phila Pa 1976) [Internet]. 2005 Jan 1 [cited 2016 Jun 19];30(1):E1-7.

26. Little P, Lewith G, Webley F, Evans M, Beattie A, Middleton K, et al. Randomised controlled trial of Alexander technique lessons, exercise, and massage (ATEAM) for chronic and recurrent back pain. Br J Sports Med [Internet]. 2008 Dec [cited 2016 Jun 19];42(12):965-8.

27. Chiu TTW, Hui-Chan CWY, Chein G. A randomized clinical trial of TENS and exercise for patients with chronic neck pain. Clin Rehabil [Internet]. 2005 Dec [cited 2016 Nov 15];19(8):850-60.

28. Medina-Mirapeix F, Escolar-Reina P, Gascón-Cánovas JJ, Montilla-Herrador J, Jimeno-Serrano FJ, Collins SM. Predictive factors of adherence to frequency and duration components in home exercise programs for neck and low back pain: an observational study. BMC Musculoskelet Disord [Internet]. 2009 [cited 2016 Jun 18];10:155.

29. Philadelphia Panel Evidence-Based Clinical Practice Guidelines. Philadelphia Panel Evidence-Based Clinical Practice Guidelines on for Neck Pain. Phys Ther [Internet]. 2001;81(10):1701-17. 
30. Nachmeson A, Johnson E, Carlson CA, Englund L, Gloossens M, van Tulder M et al. Back Pain, Neck Pain an Evidence Based Review.Stockholm. The Swedish Council on Technology Assessment in Health Care (SBU). The National Coordinating centre for Health Technology Assessment (NCCHTA). 2000;1(Report 145):1-28.

31. Iñigo V, Juste J, Ferrer M, Ortuño MA, Ruiz C, Gómez-Ferrer R. Reorientación de la actividad asistencial en un servicio de Medicina Física y Rehabilitación: análisis de costes. Evidencia y medicina coste-efectiva. Rehabilitacion [Internet]. 2010 [cited 2016 Jun 10];44(2):145-51.

32. Climent JM, Bagó J, García-López A. Patología dolorosa de columna: cervicalgia, dorsalgia y lumbalgia Definición del problema. FMC Form médica Contin en atención primaria. 2014;21:9-35.

33. Espí López L,Sentandreu Mañó G, Colorado Lluch T, Dueñas Moscardó M. Efectos de un programa de ejercicios oculocervicales en adultos en la movilidad cervical. Fisioterapia. 2011;33(2):41-9.

34. Cid J, Reig E. Cervicalgias y lumbalgias mecanicodegenerativas . Tratamiento conservador . A c t u a I i z a c i ó n. Rev la Soc Española del Dolor. 2001;8(Suplemento II):79-100.

35. Gross AR, Paquin JP, Dupont G, Blanchette S, Lalonde P, Cristie T, et al. Exercises for mechanical neck disorders: A Cochrane review update. Man Ther [Internet]. 2016 Aug [cited 2016 Nov 20];24:25-45.

Total referencias bibliográficas: 35

Referencias citadas correspondientes a RICCAFD: 0 